\title{
Pareto Points in SRAM Design Using the Sleepy Stack Approach
}

\author{
Jun Cheol Park ${ }^{1}$ and Vincent J. Mooney III $^{2}$ \\ ${ }^{1}$ Intel Corp., Folsom CA, USA \\ Juncheol.park@intel.com \\ ${ }^{2}$ Georgia Institute of Technology, Atlanta GA, USA \\ mooney@ece.gatech.edu
}

\begin{abstract}
Leakage power consumption of current CMOS technology is already a great challenge. ITRS projects that leakage power consumption may come to dominate total chip power consumption as the technology feature size shrinks. Leakage is a serious problem particularly for SRAM which occupies large transistor count in most state-of-the-art chip designs. We propose a novel ultra-low leakage SRAM design which we call "sleepy stack SRAM." Unlike the straightforward sleep approach, sleepy stack SRAM can retain logic state during sleep mode, which is crucial for a memory element. Compared to the best alternative we could find, a 6-T SRAM cell with high-Vth transistors, the sleepy stack SRAM cell with $2 \mathrm{xV}$ th at $110^{\circ} \mathrm{C}$ achieves, using $0.07 \mu$ technology models, more than $2.77 \mathrm{X}$ leakage power reduction at a cost of $16 \%$ delay increase and $113 \%$ area increase. Alternatively, by widening wordline transistors and transistors in the pull-down network, the sleepy stack SRAM cell can achieve $2.26 \mathrm{X}$ leakage reduction without increasing delay at a cost of a $125 \%$ area penalty.
\end{abstract}

\section{Introduction}

Power consumption is one of the top concerns of Very Large Scale Integration (VLSI) circuit design, for which Complementary Metal Oxide Semiconductor (CMOS) is the primary technology. Today's focus on low power is not only because of the recent growing demands of mobile applications. Even before the mobile era, power consumption has been a fundamental problem. Power consumption of CMOS consists of dynamic and static components. Although dynamic power accounted for $90 \%$ or more of the total chip power previously, as the feature size shrinks, e.g., to $0.065 \mu$ and $0.045 \mu$, static power has become a great challenge for current and future technologies. Based on the International Technology Roadmap for Semiconductors

\footnotetext{
${ }^{1}$ The first author was a Ph.D. candidate at Georgia Tech when the research reported in this paper was carried out.
}

Park, J.C., Mooney III, V.J., 2007, in IFIP International Federation for Information Processing, Volume 240, VLSI-SoC: From Systems to Silicon, eds. Reis, R., Osseiran, A., Pfleiderer, H-J., (Boston: Springer), pp. 163-177. 
(ITRS) [1], Kim et al. report that subthreshold leak-age power dissipation of a chip may exceed dynamic power dissipation at the $65 \mathrm{~nm}$ feature size [2].

One of the main reasons causing the leakage power increase is increase of subthreshold leakage power. When technology feature size scales down, supply voltage and threshold voltage also scale down. Subthreshold leakage power increases exponentially as threshold voltage decreases. Furthermore, the structure of the short channel device decreases the threshold voltage even lower. Another contributor to leakage power is gate-oxide leakage power due to the tunneling current through the gate-oxide insulator. Although gate-oxide leakage power may be comparable to subthreshold leakage power in nanoscale technology, we assume other techniques will address gate-oxide leakage; for example, high-K dielectric gate insulators may provide a solution to reduce gate-leakage [2]. Therefore, this article focuses on reducing subthreshold leakage power consumption.

Although leakage power consumption is a problem for all CMOS circuits, in this article we focus on SRAM because SRAM typically occupies large area and transistor count in a System-on-a-Chip (SoC). Furthermore, considering an embedded processor example, SRAM accounts for $60 \%$ of area and $90 \%$ of the transistor count in Intel Xscale [3], and thus may potentially consume large leakage power.

In this article, we propose the sleepy stack SRAM cell design, which is a mixture of changing the circuit structure as well as using high-Vth. The sleepy stack technique $[4,5]$ achieves greatly reduced leakage power while maintaining precise logic state in sleep mode, which may be crucial for a product spending the majority of its time in sleep or stand-by mode. Based on the sleepy stack technique, the sleepy stack SRAM cell design takes advantage of ultra-low leakage and state saving.

This article is organized as follows. In Section 2, prior work in low-leakage SRAM design is discussed. In Section 3, our sleepy stack SRAM cell design approach is proposed. In Section 4 and 5, experimental methodology and the results are presented. In Section 6, conclusions are given.

\section{Previous work}

In this section, we discuss state-of-the-art low-power memory techniques, especially SRAM and cache techniques on which our research focuses.

One easy way to reduce leakage power consumption is by adopting high-Vth transistors for all SRAM cell transistors. This solution is simple but incurs delay increase.

Azizi et al. observe that in normal programs, most of the bits in a cache are zeros. Therefore, Azizi et al. propose an Asymmetric-Cell Cache (ACC), which partially applies high-Vth transistors in an SRAM cell to save leakage power if the SRAM cell is in the zero state [6]. However, the ACC leakage power savings are quite limited in case of a benchmark which fills SRAM with mostly non-zero values.

Nii et al. propose Auto-Backgate-Controlled Multi-Threshold CMOS (ABCMTCMOS), which uses Reverse-Body Bias (RBB) to reduce leakage power 
consumption [7]. RBB increases threshold voltage without losing logic state. This increased threshold voltage reduces leakage power consumption during sleep mode. However, since the ABC-MTCMOS technique needs to charge large wells, ABCMTCMOS requires significant transition time and power consumption.

The forced stack technique achieves leakage power reduction by forcing a stack structure [9]. This technique breaks down existing transistors into two transistors and takes an advantage of the stack effect, which reduces leakage power consumption by connecting two or more turned off transistors serially. The forced stack technique can be applied to a memory element such as a register [9] or an SRAM cell [10]. However, delay increase may occur due to increased resistance, and the largest leakage savings reported under specific conditions is $90 \%(1.9 \mathrm{X})$ compared to conventional SRAM in $0.07 \mu$ technology [10].

Sleep transistors can be used for SRAM cell design. Using sleep transistors, the gated-Vdd SRAM cell blocks pull-up networks from the Vdd rail (pMOS gated-Vdd) and/or blocks pull-down networks from the Gnd rail (nMOS gated-Vdd) [11]. The gated-Vdd SRAM cell achieves low leakage power consumption from both the stack effect and high-Vth sleep transistors. However, the gated-Vdd SRAM cell [14] loses state when the sleep transistors are turned off.

Flautner et al. propose the "drowsy cache" technique that switches Vdd dynamically [12]. For short-channel devices such as $0.07 \mu$ channel length devices, leakage power increases due to Drain Induced Barrier Lowering (DIBL), thereby increasing subthreshold leakage current. The drowsy cache lowers the supply voltage during drowsy mode and suppresses leakage current using DIBL. The drowsy cache technique can retain stored data at a leakage power reduction of up to $86 \%$ [12].

Our sleepy stack SRAM cell can achieve more power savings than a high-Vth, an ACC or a drowsy cache SRAM cell. Furthermore, the sleepy stack SRAM does not require large transition time and transition power consumption unlike $\mathrm{ABC}$ MTCMOS.

\section{Approach}

We first briefly review our recently proposed low-leakage structure named "sleepy stack" in Section 3.1. Then, we explain our newly proposed "sleepy stack SRAM" in Section 3.2.

\subsection{Sleepy stack reduction}

The sleepy stack technique has a structure merging the forced stack technique and the sleep transistor technique $[4,5]$. Fig. 1 shows a sleepy stack inverter. The sleepy stack technique divides existing transistors into two transistors each typically with the same width $W_{l}$ half the size of the original single transistor's width $W_{0}$ (i.e., $W_{l}=$ $W_{0} / 2$ ), thus maintaining equivalent input capacitance. The sleepy stack inverter in Fig. 1 (a) uses $W / L=3$ for the pull-up transistors and $W / L=1.5$ for the pull-down transistors, while a conventional inverter with the same input capacitance would use 
$W / L=6$ for the pull-up transistor and $W / L=3$ for the pull-down transistor (assuming carrier mobility of NMOS is twice that of PMOS). Then sleep transistors are added in parallel to one of the transistors in each set of two stacked transistors. We use half size transistor width of the original transistor (i.e., we use $W_{0} / 2$ ) for the sleep transistor width of the sleepy stack.

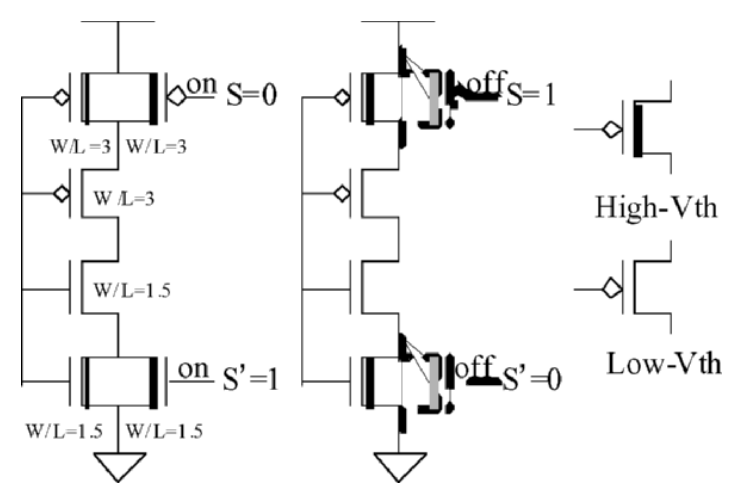

Fig. 1. (a) Sleepy stack inverter active mode (left) and (b) sleep mode (right)

During active mode, $S=0$ and $S^{\prime}=1$ are asserted, and thus all sleep transistors are turned on. This structure potentially reduces circuit delay (compared to not adding sleep transistors) because (i) added sleep transistors are always on during active mode and thus at each sleep transistor drain, the voltage value connected to a sleep transistor is always ready during active mode and (ii) there is a reduced resistance due to the two parallel transistors. Therefore, we can introduce high-Vth transistors to the sleep transistors and transistors in parallel with the sleep transistor without incurring large (e.g., $2 \mathrm{X}$ or more) delay overhead. During sleep mode, $S=1$ and $S^{\prime}=0$ are asserted, and so both of the sleep transistors are turned off. The high-Vth transistors and the stacked transistors in the sleepy stack approach sup-press leakage current. In short, using high-Vth transistors, the sleepy stack technique potentially achieves 200X leakage reduction over the forced stack technique. Furthermore, unlike the sleep transistor technique [11], the sleepy stack technique can retain exact logic state while achieving similar leakage reduction.

\subsection{Sleepy stack SRAM cell}

We design an SRAM cell based on the sleepy stack technique. The conventional 6-T SRAM cell consists of two coupled inverters and two wordline pass transistors as shown in Fig. 2. Since the sleepy stack technique can be applied to each transistor separately, the six transistors can be changed individually. However, to balance current flow (failure to do so potentially increases the risk of soft errors [10]), a symmetric design approach is used. 


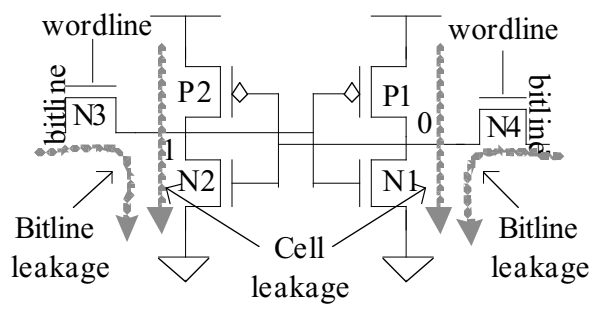

Fig. 2. SRAM cell leakage paths

Table 1. Sleepy stack applied to an SRAM cell

\begin{tabular}{|c|c|c|}
\hline Combinations & $\begin{array}{l}\text { cell leakage } \\
\text { reduction }\end{array}$ & $\begin{array}{l}\text { bitline leakage } \\
\text { reduction }\end{array}$ \\
\hline Pull-Down (PD) sleepy stack & medium & Low \\
\hline Pull-Down (PD), wordline (WL) sleepy stack & medium & High \\
\hline Pull-Up (PU), Pull-Down (PD) sleepy stack & high & Low \\
\hline $\begin{array}{l}\text { Pull-Up (PU), Pull-Down (PD), } \\
\text { wordline (WL) sleepy stack }\end{array}$ & high & high \\
\hline
\end{tabular}

There are two main types of subthreshold leakage currents in a 6-T SRAM cell: cell leakage and bitline leakage (see Fig. 2). It is very important when applying the sleepy stack technique to consider the various leakage paths in the SRAM cell. Since "Pull-Down (PD) sleepy stack" can suppress both cell leakage and bitline leakage paths together as shown in Fig. 2, we consider four combinations of the sleepy stack SRAM cell based on "Pull-Down (PD) sleepy stack" as shown in Table 1. In Table 1, "Pull-Down (PD) sleepy stack" means that the sleepy stack technique is only applied to the pull-down transistors of an SRAM cell as indicated in the bottom dashed box in Fig. 3. "Pull-Down (PD), wordline (WL) sleepy stack" means that the sleepy stack technique is applied to the pull-down transistors as well as wordline transistors. Similarly, "Pull-Up (PU), Pull-Down (PD) sleepy stack" means that the sleepy stack technique is applied to the pull-up transistors and the pull-down transistors (but not to the wordline transistors) of an SRAM cell. Finally, "Pull-Up (PU), Pull-Down (PD), wordline (WL) sleepy stack" means that the sleepy stack technique is applied to all the transistors in an SRAM cell. 


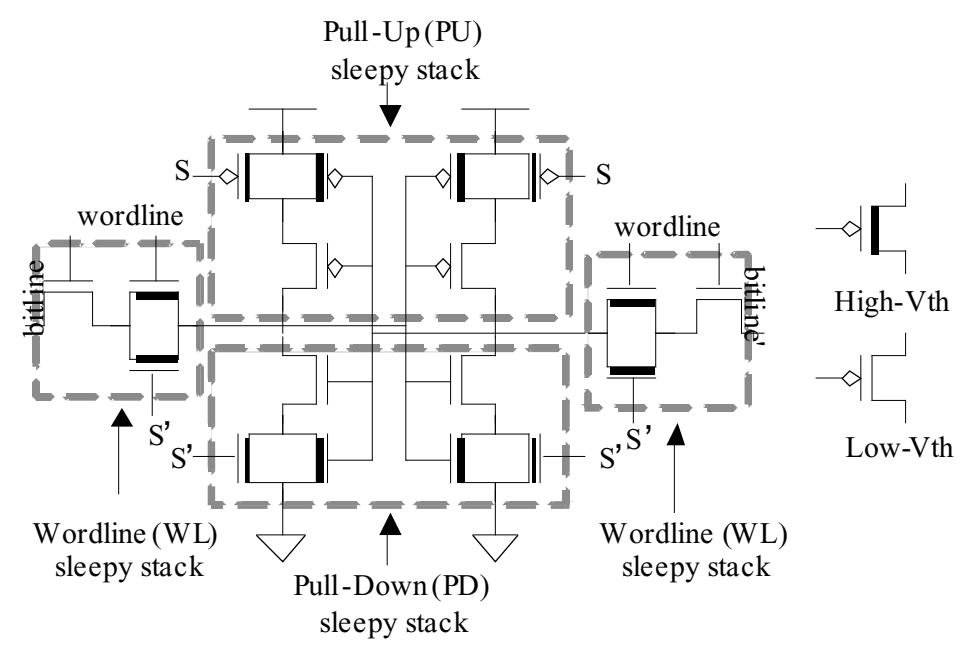

Fig. 3. Sleepy stack SRAM cell

The PD sleepy stack can suppress some part of the cell leakage. Meanwhile, the PU, PD sleepy stack can suppress the majority of the cell leakage. However, without applying the sleepy stack technique to the wordline (WL) transistors, bitline leakage cannot be significantly suppressed. Although lying in the bitline leakage path, the pull-down sleepy stack is not effective to suppress both bitline leakage paths because one of the pull-down sleepy stacks is always on. Therefore, to suppress subthreshold leakage current in a SRAM cell fully, the PU, PD and WL sleepy stack approach needs to be considered as shown in Fig. 3.

The sleepy stack SRAM cell design results in area increase because of the increase in the number of transistors. However, we halve the transistor widths in a conventional SRAM cell to make the area increase of the sleepy stack SRAM cell not necessarily directly proportional to the number of transistors. Halving a transistor width is possible when the original transistor width is at least $2 \mathrm{X}$ larger than the minimum transistor width (which is typically the case in modern high performance SRAM cell design). Unlike the conventional 6-T SRAM cell, the sleepy stack SRAM cell requires the routing of one or two extra wires for the sleep control signal(s).

\section{Experimental methodology}

To evaluate the sleepy stack SRAM cell, we compare our technique to (i) using highVth transistors as direct replacements for low-Vth transistors (thus maintaining only 6 transistors in an SRAM cell) and (ii) the forced stack technique [8]; we choose these techniques because these two techniques are state saving techniques without high risk of soft error [10]. Although Asymmetric-Cell SRAM explained in Section 2 is also a state-saving SRAM cell design, we do not consider Asymmetric-Cell 
SRAM because we assume that our SRAM cells are filled equally with ' $1 \mathrm{~s}$ ' and '0s.' This is not the condition that ACC prefers, and under this condition the leakage power savings of ACC are smaller than the high-Vth SRAM cell, which uses highVth for all six transistors.

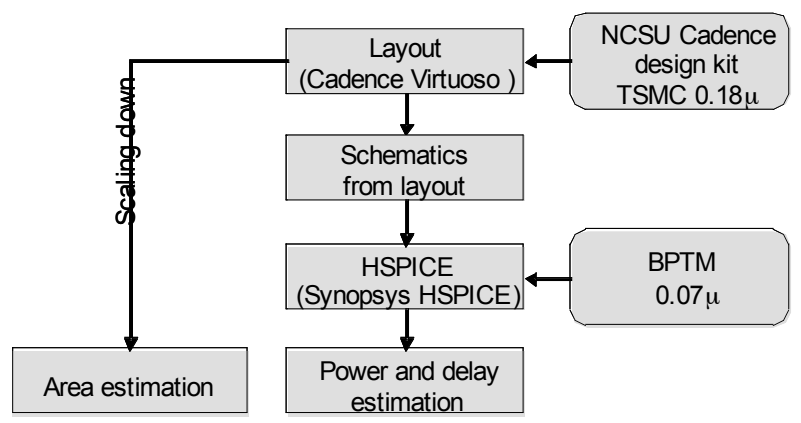

Fig. 4. Experimental procedure

Fig. 4 shows the experimental methodology used. We first layout SRAM cells of each technique. Instead of starting from scratch, we use the CACTI model for the SRAM structure and transistor sizing [13]. We use NCSU Cadence design kit targeting TSMC $0.18 \mu$ technology [14]. By scaling down the $0.18 \mu$ layout, we obtain $0.07 \mu$ technology transistor level HSPICE schematics [4], and we design a 64x64bit SRAM cell array.

We estimate area directly from our custom layout using TSMC $0.18 \mu$ technology and scale to $0.07 \mu$ using the following formula: $0.0 \mu$ area $=0.18 \mu$ area $X 0.07 \mu^{2}$ / $0.18 \mu^{2} X 1.1$ (non-linear overhead) [4]. We are aware this is not exact, hence the word "estimate." We also assume the area of the SRAM cell with high-Vth transistors is the same as with low-Vth transistors. This assumption is reasonable because high-Vth can be implemented by changing gate oxide thickness and/or channel doping levels, and this almost does not affect area at all. We estimate dynamic power, static power and read time of each of the various SRAM cell designs using HSPICE simulation with Berkeley Predictive Technology Model (BPTM) targeting $0.07 \mu$ technology [15]. The read time is measured from the time when an enabled wordline reaches $10 \%$ of the Vdd voltage to the time when either bitline or bitline' drops from $100 \%$ of the precharged voltage to $90 \%$ of the precharged voltage value while the other remains high. Therefore, one of the bitline signals remains at $\mathrm{Vdd}$, and the other is $0.9 \mathrm{xVdd}$. This $10 \%$ voltage difference between bitline and bitline' is typically enough for a sense amplifier to detect the stored cell value [6]. Dynamic power of the SRAM array is measured during the read operation with cycle time of 4ns. Static power of the SRAM cell is measured by turning off sleep transistors if applicable. To avoid leakage power measurement biased by a majority of ' 1 ' versus ' 0 ' (or vice-versa) values, half of the cells are randomly set to ' 0 ,' with the remaining half of the cells set to ' 1 .'

We compare the sleepy stack SRAM cell to the conventional 6-T SRAM cell, high-Vth 6-T SRAM cell and forced stack SRAM cell. For the "high-Vth" technique 
and the forced stack technique, we consider the same technique combinations we applied to the sleepy stack SRAM cell - see Table 1.

Table 2. Applied SRAM techniques

\begin{tabular}{lll}
\hline & Technique & Description \\
\hline Case1 & Low-Vth Std & Conventional 6T SRAM \\
Case2 & PD high-Vth & High-Vth applied to PD \\
Case3 & PD, WL high-Vth & High-Vth applied to PD, WL \\
Case4 & PU, PD high-Vth & High-Vth applied to PU, PD \\
Case5 & PU, PD, WL high-Vth & High-Vth applied to PU, PD, WL \\
Case6 & PD stack & Stack applied to PD \\
Case7 & PD, WL stack & Stack applied to PD, WL \\
Case8 & PU, PD stack & Stack applied to PU, PD \\
Case9 & PU, PD, WL stack & Stack applied to PU, PD, WL \\
Case10 & PD sleepy stack & Sleepy stack applied to PD \\
Case11 & PD, WL sleepy stack & Sleepy stack applied to PD, WL \\
Case12 & PU, PD sleepy stack & Sleepy stack applied to PU, PD \\
Case13 & PU, PD, WL sleepy stack & Sleepy stack applied to PU, PD, WL \\
\hline
\end{tabular}

To properly observe the techniques, we compare 13 different cases as shown in Table 2. Case1 is the conventional 6-T SRAM cell, which is our base case. Cases 2, 3, 4 and 5 are 6-T SRAM cells using the high-Vth technique. PD high-Vth is the high-Vth technique applied only to the pull-down transistors. PD, WL high-Vth is the high-Vth technique applied to the pull-down transistors as well as to the wordline transistors. PU, PD high-Vth is the high-Vth technique applied to the pullup and pull-down transistors. PU, PD, WL high-Vth is the high-Vth technique applied to all the SRAM transistors. Cases 6, 7,8 and 9 are 6-T SRAM cells with the forced stack technique [8]. PD stack is the forced stack technique applied only to the pull-down transistors. PD, WL stack is the forced stack technique applied to the pull-down transistors as well as to the wordline transistors. PU, PD stack is the forced stack technique applied to the pull-up and pull-down transistors. PU, PD, WL stack is the forced stack technique applied to all the SRAM transistors. Please note that we do not apply high-Vth to the forced stack technique because the forced stack SRAM with high-Vth incurs more than $2 \mathrm{X}$ de-lay increase. Cases 10, 11, 12 and 13 are the four sleepy stack SRAM cell approaches as listed in Table 1. For sleepy stack SRAM, high-Vth is applied only to the sleep transistors and the transistors parallel to the sleep transistors as shown in Fig. 3.

\section{Results}

In this section, we explore the experimental results for the different sleepy stack SRAM cell variations. We consider area, cell read time, leakage power, active 
power. Then we discuss tradeoffs in leakage power techniques followed by static noise margin, which represents the noise immunity of SRAM.

\subsection{Area}

Table 3. Area

\begin{tabular}{lllllll}
\hline & Technique & $\begin{array}{l}\text { Height } \\
{[\mu]}\end{array}$ & $\begin{array}{l}\text { Width } \\
{[\mu]}\end{array}$ & $\begin{array}{l}0.18 \mathrm{u} \text { tech. } \\
\text { Area }\left[\mu^{2}\right]\end{array}$ & $\begin{array}{l}0.07 \mathrm{u} \text { tech. } \\
\text { Area }\left[\mu^{2}\right]\end{array}$ & $\begin{array}{l}\text { Normalized } \\
\text { area }\end{array}$ \\
\hline Case1 & Low-Vth Std & 3.825 & 4.500 & 17.213 & 2.864 & 1.00 \\
Case2 & PD high-Vth & 3.825 & 4.500 & 17.213 & 2.864 & 1.00 \\
Case3 & PD, WL high-Vth & 3.825 & 4.500 & 17.213 & 2.864 & 1.00 \\
Case4 & PU, PD high-Vth & 3.825 & 4.500 & 17.213 & 2.864 & 1.00 \\
Case5 & PU, PD, WL high-Vth & 3.825 & 4.500 & 17.213 & 2.864 & 1.00 \\
Case6 & PD stack & 3.465 & 4.680 & 16.216 & 2.698 & 0.94 \\
Case7 & PD, WL stack & 3.465 & 5.760 & 19.958 & 3.320 & 1.16 \\
Case8 & PU, PD stack & 3.285 & 4.680 & 15.374 & 2.558 & 0.89 \\
Case9 & PU, PD, WL stack & 3.465 & 5.760 & 19.958 & 3.320 & 1.16 \\
Case10 & PD sleepy stack & 4.545 & 5.040 & 22.907 & 3.811 & 1.33 \\
Case11 & PD, WL sleepy stack & 4.455 & 6.705 & 29.871 & 4.969 & 1.74 \\
Case12 & PU, PD sleepy stack & 5.760 & 5.040 & 29.030 & 4.829 & 1.69 \\
Case13 & PU, PD, WL sleepy & 5.535 & 6.615 & 36.614 & 6.091 & 2.13 \\
& stack & & & & & \\
\hline
\end{tabular}

Table 3 shows the area of each technique. Please note that SRAM cell area can be reduced further by using minimum size transistors, but reducing transistor size increases cell read time. Some SRAM cells with the forced stack technique show smaller area even compared to the base case. The reason is that divided transistors can enable a particularly squeezed design [4]. The sleepy stack technique increases area by between $33 \%$ and $113 \%$. The added sleep transistors are a bottleneck to reduce the size of the sleepy stack SRAM cells. Further, wiring the sleep control signals (an overhead we do not consider in Table 3) makes the design more complicated.

\subsection{Cell read time}

Although SRAM cell read time changes slightly as temperature changes, the impact of temperature on the cell read time is quite small. However, the impact of threshold voltage is large. We apply $1.5 \mathrm{xV}$ th and $2 \mathrm{xV}$ th for the high-Vth technique and the sleepy stack technique. As shown in Table 4, the delay penalty of the forced stack technique (with all low-Vth transistors) is between $35 \%$ and $70 \%$ compared to the standard 6-T SRAM cell. This is one of the primary reasons that the forced stack technique cannot use high-Vth transistors without incurring dramatic delay increase (e.g., $2 \mathrm{X}$ or more delay penalty is observed using either $1.5 \mathrm{xV}$ th or $2 \mathrm{xV}$ th). 
Table 4. Normalized cell read time (absolute numbers available in [4])

\begin{tabular}{llcccccc}
\hline & Technique & \multicolumn{3}{c}{$25^{\circ} \mathrm{C}$} & \multicolumn{3}{c}{$110^{\circ} \mathrm{C}$} \\
\hline & & $1 \times V t h$ & $1.5 x V t h$ & $2 x V t h$ & $1 x V t h$ & $1.5 x V t h$ & $2 x V t h$ \\
Case1 & Low-Vth Std & 1.000 & N/A & N/A & 1.000 & N/A & N/A \\
Case2 & PD high-Vth & N/A & 1.022 & 1.043 & N/A & 1.020 & 1.061 \\
Case3 & PD, WL high-Vth & N/A & 1.111 & 1.280 & N/A & 1.117 & 1.262 \\
Case4 & PU, PD high-Vth & N/A & 1.022 & 1.055 & N/A & 1.020 & 1.048 \\
Case5 & PU, PD, WL high-Vth & N/A & 1.111 & 1.277 & N/A & 1.110 & 1.259 \\
Case6 & PD stack & 1.368 & N/A & N/A & 1.345 & N/A & N/A \\
Case7 & PD, WL stack & 1.647 & N/A & N/A & 1.682 & N/A & N/A \\
Case8 & PU, PD stack & 1.348 & N/A & N/A & 1.341 & N/A & N/A \\
Case9 & PU, PD, WL stack & 1.704 & N/A & N/A & 1.678 & N/A & N/A \\
Case10 & PD sleepy stack & N/A & 1.276 & 1.307 & N/A & 1.263 & 1.254 \\
Case11 & PD, WL sleepy stack & N/A & 1.458 & 1.551 & N/A & 1.435 & 1.546 \\
Case12 & PU, PD sleepy stack & N/A & 1.275 & 1.306 & N/A & 1.287 & 1.319 \\
Case13 & PU, PD, WL sleepy & N/A & 1.456 & 1.605 & N/A & 1.450 & 1.504 \\
\hline
\end{tabular}

Among the three low-leakage techniques, the sleepy stack technique is the second best in terms of cell read time. The PU, PD, WL high-Vth with $2 x V$ th is $16 \%$ faster than the PU, PD, WL sleepy stack with $2 x V$ th at $110^{\circ} \mathrm{C}$. Since we are aware that area and delay are critical factors when designing SRAM, we will explore area and delay impact using tradeoffs in Section 5.4. However, let us first discuss leakage reduction.

\subsection{Leakage power}

We measure leakage power while changing threshold voltage and temperature because the impact of threshold voltage and temperature on leakage power is significant. Table 5 shows leakage power consumption with two high-Vth values, $1.5 \mathrm{xV}$ th and $2 \mathrm{xV}$ th, and two temperatures, $25^{\circ} \mathrm{C}$ and $110^{\circ} \mathrm{C}$, where Case 1 and the cases using the forced stack technique (Cases 6, 7,8 and 9) are not affected by changing Vth because these use only low-Vth. (Please note the absolute numbers are available in [12].)

5.3.1 Results at $25^{\circ} \mathrm{C}$ Our results at $25^{\circ} \mathrm{C}$ show that Case 5 is the best with $2 \mathrm{xV}$ th and Case 13 is the best with $1.5 \mathrm{xV}$ th. Specially, at $1.5 \mathrm{xV}$ th, Case 5 and Case 13 achieve $25 \mathrm{X}$ and $60 \mathrm{X}$ leakage reduction over Case1, respectively. However, the leakage reduction comes with delay increase. The delay penalty is $11 \%$ and $45 \%$, respectively, compared to Case 1 . 
Table 5. Normalized leakage power (absolute numbers available in [4])

\begin{tabular}{|c|c|c|c|c|c|c|c|}
\hline & \multirow[t]{2}{*}{ Technique } & \multicolumn{3}{|c|}{$25^{\circ} \mathrm{C}$} & \multicolumn{3}{|c|}{$110^{\circ} \mathrm{C}$} \\
\hline & & $1 \mathrm{xVth}$ & $1.5 \mathrm{xVth}$ & $2 \mathrm{xVth}$ & $1 \mathrm{xVth}$ & $1.5 \mathrm{xVth}$ & $2 \mathrm{xVth}$ \\
\hline Case1 & Low-Vth Std & 1.0000 & $\mathrm{~N} / \mathrm{A}$ & N/A & 1.0000 & $\mathrm{~N} / \mathrm{A}$ & $\mathrm{N} / \mathrm{A}$ \\
\hline Case2 & PD high-Vth & N/A & 0.5466 & 0.5274 & $\mathrm{~N} / \mathrm{A}$ & 0.5711 & 0.5305 \\
\hline Case3 & PD, WL high-Vth & N/A & 0.2071 & 0.1736 & $\mathrm{~N} / \mathrm{A}$ & 0.2555 & 0.1860 \\
\hline Case4 & PU, PD high-Vth & N/A & 0.3785 & 0.3552 & $\mathrm{~N} / \mathrm{A}$ & 0.4022 & 0.3522 \\
\hline Case 5 & $\begin{array}{l}\text { PU, PD, WL high- } \\
\text { Vth }\end{array}$ & $\mathrm{N} / \mathrm{A}$ & 0.0391 & 0.0014 & N/A & 0.0857 & 0.0065 \\
\hline Case6 & PD stack & 0.5541 & N/A & N/A & 0.5641 & N/A & N/A \\
\hline Case 7 & PD, WL stack & 0.2213 & $\mathrm{~N} / \mathrm{A}$ & N/A & 0.2554 & $\mathrm{~N} / \mathrm{A}$ & $\mathrm{N} / \mathrm{A}$ \\
\hline Case 8 & PU, PD stack & 0.3862 & $\mathrm{~N} / \mathrm{A}$ & N/A & 0.3950 & N/A & N/A \\
\hline Case9 & PU, PD, WL stack & 0.0555 & $\mathrm{~N} / \mathrm{A}$ & N/A & 0.0832 & N/A & $\mathrm{N} / \mathrm{A}$ \\
\hline Case10 & PD sleepy stack & $\mathrm{N} / \mathrm{A}$ & 0.5331 & 0.5315 & $\mathrm{~N} / \mathrm{A}$ & 0.5282 & 0.5192 \\
\hline Case11 & PD, WL sleepy stack & N/A & 0.1852 & 0.1827 & $\mathrm{~N} / \mathrm{A}$ & 0.1955 & 0.1820 \\
\hline Case12 & PU, PD sleepy stack & $\mathrm{N} / \mathrm{A}$ & 0.3646 & 0.3630 & $\mathrm{~N} / \mathrm{A}$ & 0.3534 & 0.3439 \\
\hline Case13 & $\begin{array}{l}\text { PU, PD, WL sleepy } \\
\text { stack }\end{array}$ & N/A & 0.0167 & 0.0033 & $\mathrm{~N} / \mathrm{A}$ & 0.0167 & 0.0024 \\
\hline
\end{tabular}

5.3.2 Results at $110^{\circ} \mathrm{C}$ Absolute power consumption numbers at $110^{\circ} \mathrm{C}$ show more than $10 \mathrm{X}$ increase of leakage power consumption compared to the results at $25^{\circ} \mathrm{C}$. This could be a serious problem for SRAM because SRAM often resides next to a microprocessor whose temperature is high.

At $110^{\circ} \mathrm{C}$, the sleepy stack technique shows the best result in both $1.5 \mathrm{xV}$ th and $2 \mathrm{xVth}$ even compared to the high-Vth technique. The leakage performance degradation under high temperature is very noticeable with the high-Vth technique and the forced stack technique. For example, at $25^{\circ} \mathrm{C}$ the high-Vth technique with $1.5 \mathrm{xV}$ th (Case5) and the forced stack technique (Case9) show around 96\% leakage reduction. However, at $110^{\circ} \mathrm{C}$ the same techniques show around $91 \%$ of leakage power reduction compared to Case1. Only the sleepy stack technique achieves superior leakage power reduction; after increasing temperature, the sleepy stack SRAM shows 5.1X and 4.8X reductions compared to Case5 and Case9, respectively, with $1.5 \mathrm{xV}$ th.

When the low-leakage techniques are applied only to the pull-up and pull-down transistors, leakage power reduction is at most $65 \%\left(2 \times \mathrm{Vth}, 110^{\circ} \mathrm{C}\right)$ because bitline leakage cannot be suppressed. The remaining $35 \%$ of leakage power can be suppressed by applying low-leakage techniques to wordline transistors. This implies that bitline leakage power addresses around 35\% of SRAM cell leakage power consumption. This trend is observed for all three techniques considered, i.e., highVth, forced stack and sleepy stack.

\subsection{Tradeoffs in low-leakage techniques}

Although the sleepy stack technique shows superior results in terms of leakage power, we need to explore area, delay and power together because the sleepy stack technique comes with non-negligible area and delay penalties. To be compared with 
the high-Vth technique at the same cell read time, we consider four more cases for sleepy stack SRAM in addition to the cases already considered in Table 5; we increase the widths of all wordline and pull-down transistors (including sleep transistors). Specifically, for the sleepy stack technique, we find new transistor widths of wordline transistors and pull-down transistors such that the result is delay approximately equal to the delay of the 6-T high-Vth case, i.e., Case5. The new cases are marked with '*' (Cases $10^{*}, 11 *, 12 *, 13 *$ ). The results are shown in Table 6. To enhance readability of tradeoffs, each table is sorted by leakage power. Although we compared four different simulation conditions, we take the condition with $2 \mathrm{xV}$ th at $110^{\circ} \mathrm{C}$ as important representative technology points at which to compare the trade-offs between techniques. We choose $110^{\circ} \mathrm{C}$ because generally SRAM operates at a high temperature and also because high temperature is the "worst case."

In Table 6, we observe six Pareto points, respectively, which are in shaded rows, considering three variables of leakage, delay, and area. Case13 shows the lowest possible leakage, 2.7X smaller than the leakage of any of the prior approaches considered; however, there is a corresponding delay and area penalty. Alternatively, Case $13 *$ shows the same delay (within $0.2 \%$ ) as Case 5 and $2.26 \mathrm{X}$ leakage reduction over Case 5; however, Case $13^{*}$ uses $125 \%$ more area than Case 5 . In short, this article presents new, previously unknown Pareto points at the low-leakage end of the spectrum (for a definition of a "Pareto point" please see [16]).

Table 6. Tradeoffs $\left(2 x V t h, 110^{\circ} \mathrm{C}\right)$

\begin{tabular}{llccc}
\hline Technique & $\begin{array}{c}\text { Normalized } \\
\text { leakage }\end{array}$ & $\begin{array}{c}\text { Normalized } \\
\text { delay }\end{array}$ & $\begin{array}{c}\text { Normalized } \\
\text { area }\end{array}$ \\
\hline Case1 & Low-Vth Std & 1.000 & 1.000 & 1.000 \\
Case6 & PD stack & 0.564 & 1.345 & 0.942 \\
Case2 & PD high-Vth & 0.530 & 1.061 & 1.000 \\
Case10 & PD sleepy stack & 0.519 & 1.254 & 1.331 \\
Case10* & PD sleepy stack* & 0.519 & 1.254 & 1.331 \\
Case8 & PU, PD stack & 0.395 & 1.341 & 0.893 \\
Case4 & PU, PD high-Vth & 0.352 & 1.048 & 1.000 \\
Case12* & PU, PD sleepy stack* & 0.344 & 1.270 & 1.713 \\
Case12 & PU, PD sleepy stack & 0.344 & 1.319 & 1.687 \\
Case7 & PD, WL stack & 0.255 & 1.682 & 1.159 \\
Case3 & PD, WL high-Vth & 0.186 & 1.262 & 1.000 \\
Case11* & PD, WL sleepy stack* & 0.183 & 1.239 & 1.876 \\
Case11 & PD, WL sleepy stack & 0.182 & 1.546 & 1.735 \\
Case9 & PU, PD, WL stack & 0.083 & 1.678 & 1.159 \\
Case5 & PU, PD, WL high-Vth & 0.007 & 1.259 & 1.000 \\
Case13* & PU, PD, WL sleepy stack* & 0.003 & 1.265 & 2.253 \\
Case13 & PU, PD, WL sleepy stack & 0.002 & 1.504 & 2.127 \\
\hline
\end{tabular}




\subsection{Active power}

Table 7 shows power consumption during read operations. The active power consumption includes dynamic power used to charge and discharge SRAM cells plus leakage power consumption. At $25^{\circ} \mathrm{C}$ leakage power is less than $20 \%$ of the active power in case of the standard low-Vth SRAM cell in $0.07 \mathrm{u}$ technology according to BPTM [15]. However, leakage power increases $10 \mathrm{X}$ as the temperature changes to $110^{\circ} \mathrm{C}$ although active power increases $3 \mathrm{X}$. At $110^{\circ} \mathrm{C}$, leakage power is more than half of the active power from our simulation results. Therefore, without an effective leakage power reduction technique, total power consumption - even in active mode is affected significantly.

Table 7. Normalized active power (absolute numbers available in [4])

\begin{tabular}{|c|c|c|c|c|c|c|c|}
\hline & \multirow[t]{2}{*}{ Technique } & \multicolumn{3}{|c|}{$25^{\circ} \mathrm{C}$} & \multicolumn{3}{|c|}{$110^{\circ} \mathrm{C}$} \\
\hline & & $1 \mathrm{xV}$ th & $1.5 \mathrm{xVth}$ & $2 \mathrm{xVth}$ & $1 \mathrm{xVth}$ & $1.5 \times \mathrm{Xth}$ & $2 \mathrm{xVth}$ \\
\hline Case 1 & Low-Vth Std & 1.000 & N/A & N/A & 1.000 & N/A & N/A \\
\hline Case2 & PD high-Vth & N/A & 0.936 & 0.913 & N/A & 0.724 & 0.691 \\
\hline Case 3 & PD, WL high-Vth & N/A & 0.858 & 0.829 & N/A & 0.618 & 0.478 \\
\hline Case4 & PU, PD high-Vth & N/A & 0.928 & 0.893 & N/A & 0.572 & 0.582 \\
\hline Case 5 & $\begin{array}{l}\text { PU, PD, WL high- } \\
\text { Vth }\end{array}$ & N/A & 0.838 & 0.842 & N/A & 0.432 & 0.368 \\
\hline Case6 & PD stack & 0.926 & N/A & N/A & 0.669 & N/A & N/A \\
\hline Case 7 & PD, WL stack & 0.665 & N/A & N/A & 0.398 & N/A & N/A \\
\hline Case8 & PU, PD stack & 0.905 & N/A & N/A & 0.596 & N/A & N/A \\
\hline Case9 & PU, PD, WL stack & 0.637 & N/A & N/A & 0.293 & N/A & N/A \\
\hline Case10 & PD sleepy stack & N/A & 0.981 & 0.981 & N/A & 0.807 & 0.811 \\
\hline Case11 & PD, WL sleepy stack & N/A & 0.773 & 0.717 & N/A & 0.586 & 0.600 \\
\hline Case12 & PU, PD sleepy stack & N/A & 0.961 & 1.005 & N/A & 0.786 & 0.797 \\
\hline Case13 & $\begin{array}{l}\text { PU, PD, WL sleepy } \\
\text { stack }\end{array}$ & N/A & 0.719 & 0.708 & N/A & 0.588 & 0.546 \\
\hline
\end{tabular}

\subsection{Static noise margin}

Changing the SRAM cell structure may change the static noise immunity of the SRAM cell. Thus, we measure the Static Noise Margin (SNM) of the sleepy stack SRAM cell and the conventional 6-T SRAM cell. The SNM is defined by the size of the maximum nested square in a butterfly plot. The SNM of the sleepy stack SRAM cell is measured twice in active mode and sleep mode, and the results are shown in Table 8. The SNM of the sleepy stack SRAM cell in active mode is $0.299 \mathrm{~V}$ and almost exactly the same as the SNM of a conventional SRAM cell; the SNM of a conventional SRAM cell is $0.299 \mathrm{~V}$. Although we do not perform a process variation analysis, we expect that the high SNM of the sleepy stack SRAM cell makes the technique as immune to process variations as a conventional SRAM cell. 
Table 8. Static noise margin

\begin{tabular}{llcc}
\hline & Technique & Active mode & Sleep mode \\
\hline Case1 & Low-Vth Std & 0.299 & N/A \\
Case10 & PD sleepy stack & 0.317 & 0.362 \\
Case11 & PD, WL sleepy stack & 0.324 & 0.363 \\
Case12 & PU, PD sleepy stack & 0.299 & 0.384 \\
Case13 & PU, PD, WL sleepy stack & 0.299 & 0.384 \\
\hline
\end{tabular}

\section{Conclusions}

In this article, we have presented and evaluated our newly proposed "sleepy stack SRAM" Our sleepy stack SRAM provides the largest leakage savings among all alternatives considered. Specifically, compared to a standard SRAM cell - Case1 Table 5 shows that at $110^{\circ} \mathrm{C}$ and $2 \mathrm{xV}$ th, Case 13 reduces leakage by $424 \mathrm{X}$ as compared to Case1; unfortunately, this $424 \mathrm{X}$ reduction comes as a cost of a delay increase of $50.4 \%$ and an area penalty of $113 \%$. Resizing the sleepy stack SRAM can reduce delay significantly at a cost of less leakage savings; specifically, Case 13* is an interesting Pareto point as discussed in Section 5.4.

We believe that this article presents an important development because our sleepy stack SRAM seems to provide, in general, the lowest leakage Pareto points of any VLSI design style known to the authors. Given the nontrivial area penalty (e.g., up to $125 \%$ for Case $13 *$ in Table 6), perhaps sleepy stack SRAM would be most appropriate for a small SRAM intended to store minimal standby data for an embedded system spending significant time in standby mode; for such a small SRAM (e.g., 16KB), the area penalty may be acceptable given system-level standby power requirements. If absolute minimum leakage power is extremely critical, then perhaps specific target embedded systems could use sleepy stack SRAM more widely.

\section{Reference}

1. International Technology Roadmap for Semiconductors by Semiconductor Industry Association (2002).

2. N. S. Kim, T. Austin, D. Baauw, T. Mudge, K. Flautner, J. Hu, M. Irwin, M. Kandemir, V. Narayanan, Leakage Current: Moore's Law Meets Static Power, IEEE Computer 36(12), pp. 68-75 (2003).

3. L. Clark, E. Hoffman, J. Miller, M. Biyani, L. Luyun, S. Strazdus, M. Morrow, K. Velarde, M. Yarch, An Embedded 32-b Microprocessor Core for Low-Power and High-Performance Applications, IEEE Journal of Solid-State Circuits 36(11), 1599-1608 (2001).

4. J. Park, Sleepy Stack: a New Approach to Low Power VLSI and Memory, Ph.D. dissertation, School of Electrical and Computer Engineering, Georgia Institute of Technology, 2005. 
5. J. Park, V. J. Mooney, P. Pfeiffenberger, Sleepy Stack Reduction in Leak-age Power, Proceedings of the International Workshop on Power and Timing Modeling, Optimization and Simulation (PATMOS'04), pp. 148-158 (2004).

6. N. Azizi, A. Moshovos, F. Najm, Low-Leakage Asymmetric-Cell SRAM, Proceedings of the International Symposium on Low Power Electronics and Design, pp. 48-51 (2002).

7. K. Nii, H. Makino, Y. Tujihashi, C. Morishima, Y. Hayakawa, H. Nunogami, T. Arakawa, H. Hamano, A Low Power SRAM Using Auto-Backgate-Controlled MT-CMOS, Proceedings of the International Symposium on Low Power Electronics and Design, pp. 293-298 (1998).

8. S. Narendra, V. D. S. Borkar, D. Antoniadis, A. Chandrakasan, Scaling of Stack Effect and its Application for Leakage Reduction, Proceedings of the International Symposium on Low Power Electronics and Design, pp. 195-200 (2001).

9. S. Tang, S. Hsu, Y. Ye, J. Tschanz, D. Somasekhar, S. Narendra, S. L. Lu, R. Krishnamurthy, V. De, Scaling of Stack Effect and its Application for Leakage Reduction, Symposium on VLSI Circuits Digest of Technical Papers, pp. 320-321 (2002).

10. V. Degalahal, N. Vijaykrishnan, M. Irwin, Analyzing soft errors in leakage optimized SRAM design, IEEE International Conference on VLSI Design, pp. 227-233 (2003).

11. M. Powell, S. H. Yang, B. Falsafi, K. Roy, T. N. Vijaykumar, Gated-Vdd: A Circuit Technique to Reduce Leakage in Deep-submicron Cache Memories, Proceedings of the International Symposium on Low Power Electronics and Design, pp. 90-95 (2000).

12. K. Flautner, N. S. Kim, S. Martin, D. Blaauw, T. Mudge, Drowsy Caches: Simple Techniques for Reducing Leakage Power, Proceedings of the International Symposium on Computer Architecture, pp. 148-157 (2002).

13. S. Wilton, N. Jouppi, An Enhanced Access and Cycle Time Model for On-Chip Caches (1993); http://www.hpl.hp.com/techreports/Compaq-DEC/WRL-93-5.pdf.

14. NC State University Cadence Tool Information; http://www.cadence.ncsu.edu.

15. Berkeley Predictive Technology Model (BPTM); http://www.eas.asu.edu/ ptm/.

16. G. De Micheli, Synthesis and Optimization of Digital Circuits (McGraw-Hill Inc., USA, 1994). 